\title{
Onset
}

\section{MARK JARMAN}

Where did your love for them come from?

Where did they come from themselves?

The party goes on while you wash up and their conversation dissolves.

Perhaps memory's turned hard of hearing. That's a way to think of it, sure. In the impervious chatter they say frankly there is no cure.

You may catch it, then you'll forget it, but not the important things, your spouse, your children, their lovers, the happiness each of them brings.

When you are with them keep smiling.

So far the impairment is mild.

Or so you've been told in all candor.

Yes, so you've been told. 